\title{
MINIMISING COMPLEXITY IN ITERATIVE MULTIUSER DETECTION USING DYNAMIC DECODING SCHEDULES
}

\author{
David P. Shepherd ${ }^{1}$, Fredrik Brännström ${ }^{2}$, Zhenning Shi ${ }^{3}$, and Mark C. Reed ${ }^{4}$ \\ ${ }^{1,3,4}$ Wireless Signal Processing \\ Australian National University/NICTA \\ Locked Bag 8001 Canberra ACT 2601, Australia \\ ${ }^{2}$ Dept. of Signals and Systems \\ Chalmers University of Technology \\ 41296 Göteborg, Sweden
}

\begin{abstract}
In this paper we utilize extrinsic information transfer (EXIT) chart analy sis with unequal user power levels in a coded directsequence code-division multiple-access (DS-CDMA) multiuser receiver to derive a dynamic decoding schedule. Conventional receivers generally follow a static predetermined decoding schedule. However, decoding delay and complexity can be significantly reduced while maintaining BER performance through a dynamic decoding schedule optimized for a given load and transmit power configuration. Verified through simulations, we show that complexity savings of up to $50 \%$ can be achieved. We also derive the effective EXIT charts which can be used for the convergence analysis and performance predictions of unequal power CDMA systems.
\end{abstract}

\section{INTRODUCTION}

Predicting the performance of a code-division multiple-access (CDMA) system with turbo coding is computationally demanding even for a small number of users. Extrinsic information transfer (EXIT) chart analysis has been successfully used for describing and visualizing the convergence behavior without the need for simulations. However, most EXIT chart analysis of coded CDMA has focused on the fixed-schedule equal power case [1,2]. Caire et al used density evolution to optimise transmit power in an unequal power CDMA system in [3]. Variance transfer charts were used for analysis of unequal power coded CDMA in [4]. We consider an EXIT chart analysis of a turbo coded CDMA system with users transmitting at arbitrary power levels.

Iterative multiuser detection (IMUD) significantly reduces multiple access interference (MAI). Conventional IMUD receivers follow a fixed (static) decoding schedule, which can be inefficient since it potentially adds more complexity and delay to the iterative decoding process than necessary. In [5] a technique for deriving the optimal schedule for decoding of an arbitrary number of concatenated codes is described.
The method utilizes a trellis-based approach with a modified Viterbi search algorithm to find the decoding schedule to achieve convergence with the minimum total decoding complexity or number of decoder iterations. We use the results in [4] and [6] to derive the EXIT charts for an unequal power IMUD receiver and the algorithm in [5] to dynamically find the schedule which achieves a target (otherwise lowest possible) BER with the lowest decoding complexity. While [5] optimises the activation schedule for a single-user system with multiple concatenated codes, we derive the optimal schedule for multiple users with identical turbo codes and arbitrary power levels.

\section{SYSTEM DESCRIPTION}

Consider a CDMA system in which there are $K$ users generating binary information sequences which are turbo encoded, interleaved and mapped onto BPSK symbols before being spread by $K$ unique direct sequence spreaders. The turbo code is 3GPP compliant, common for all users and consists of symmetric parallel concatenated 8-state convolutional codes with generator polynomial $\left(G_{r}, G\right)=(015,013)$. The trellis is terminated in the encoders and the overall code rate is $R=1 / 3$ (no puncturing) and information block lengths range from 1296 up to 3856 bits [7].

The IMUD receiver consists of an interference canceller (IC) and $K$ turbo decoders (TDs) as shown in Fig. 1 and was first described for convolutional codes in [8]. See [9] for a good description of the turbo decoder. The interference canceller takes channel values $Y$ and $a$ priori input $a_{k}^{\mathrm{IC}}$ (from each of the $K$ users $k=1,2, \ldots, K$ ) and outputs extrinsic information (on the coded bits for each user) $E_{k}^{\mathrm{IC}}$ which is de-interleaved and becomes the a priori input $A_{k}^{\mathrm{TD}}$ to the TD for user $k$. On the first iteration of the receiver the a priori input to the interference canceller is zero. Each of the $K$ TDs outputs extrinsic information (on the coded bits) $E_{k}^{\mathrm{TD}}$ and $a$ posteriori output (on the information bits) $D_{k}^{\mathrm{TD}} \cdot E_{k}^{\mathrm{TD}}$ is inter- 


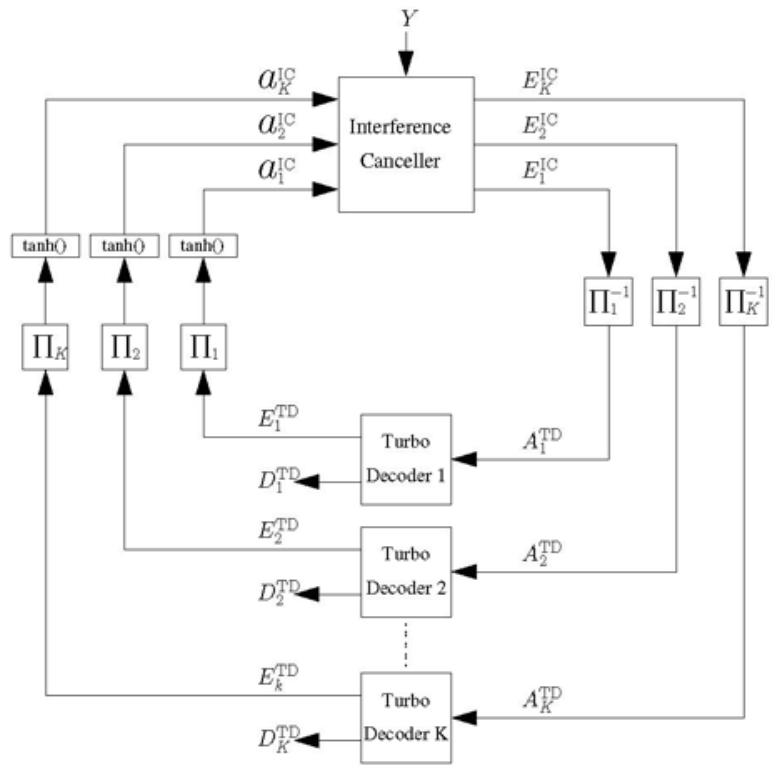

Fig. 1. IMUD receiver with interference canceller and turbo decoders.

leaved and converted to soft bits $a_{k}^{\mathrm{IC}}=\tanh \left(E_{k}^{\mathrm{TD}} / 2\right)$. Hard decisions are made on $D_{k}^{\mathrm{TD}}$.

\section{EXIT CHART ANALYSIS}

\subsection{EXIT Function}

For the equal power case the EXIT function for an interference canceller is a function of the a priori input mutual information, $I_{A}^{\mathrm{IC}}$, and the channel signal-to-noise ratio (SNR), $E_{b} / N_{0}$. The average extrinsic mutual information from the IC can therefore be described as

$$
I_{E}^{\mathrm{IC}}=T_{\mathrm{mud}}\left(I_{A}^{\mathrm{IC}}, E_{b} / N_{0}\right) .
$$

For the turbo decode TD the average extrinsic mutual information is a function of only the mutual information of the $a$ priori input, $I_{A}^{\mathrm{TD}}$,

$$
I_{E}^{\mathrm{TD}}=T_{\mathrm{dec}}\left(I_{A}^{\mathrm{TD}}\right) .
$$

In an unequal power CDMA system the users are grouped according to their power level. We assume all users within a power group are essentially identical and we therefore consider each group as a (virtual) single user. For convergence analysis, the traditional EXIT charts need to be adjusted to reflect the behavior of the system under the unequal power conditions.

\subsection{Effective EXIT Function}

Consider a CDMA system with $L$ groups of different power levels. Define $\mathbf{K}=\left[K_{1}, K_{2}, \ldots K_{L}\right]$ and $\mathbf{P}=\left[P_{1}, P_{2}, \ldots, P_{L}\right]$, where $K_{k}$ and $P_{k}$ are the number of users in the group and their transmission power, respectively, for $k=1,2, \ldots, L$. Using the results in [4] and [6], we derive effective EXIT functions. For the interference canceller, the effective EXIT function is

$$
I_{E, \text { eff }}^{\mathrm{IC}}=J\left(\sqrt{\frac{4}{\left(1-T^{-1}\left(I_{A, \mathrm{eff}}^{\mathrm{IC}}\right)\right) \frac{K_{\mathrm{eff}}-1}{N}+\frac{N_{0}}{2 R P_{\text {ref }}}}}\right)
$$

where $J(\cdot)$ is the $J$ function from [9], $I_{A \text {,eff }}^{\mathrm{IC}}$ is the effective prior mutual information for the IC, $K_{\text {eff }}=\frac{1}{P_{\text {ref }}} \sum_{k=1}^{L} K_{k} P_{k}$ is the effective number of users, $P_{\text {ref }}$ is some arbitrary reference power level, $\mathrm{N}$ is the processing gain, $R$ is the code rate and $N_{0}$ is the noise spectral density.

We generate the EXIT chart for the TD using Monte Carlo simulation. The effective EXIT function for group $k$ is then

$$
I_{E, k}^{\mathrm{TD}}=T_{\mathrm{dec}}\left(J\left(\sqrt{\frac{P_{k}}{P_{\mathrm{ref}}}} J^{-1}\left(I_{A, \text { eff }}^{\mathrm{TD}}\right)\right)\right),
$$

where $I_{A \text {,eff }}^{\mathrm{TD}}=I_{E \text {,eff }}^{\mathrm{IC}}$ is the effective prior mutual information for the TDs. The effective symbol variance $\sigma_{d \text {,eff }}^{2}$ is the sum of symbol variances $\sigma_{d, k}^{2}$ scaled by the factor [4]

$$
\alpha_{k}^{*}=\frac{K_{k} P_{k}}{K_{\text {eff }} P_{\text {ref }}} .
$$

Using the expression derived in [6], describing the relationship between symbol variance and mutual information,

$$
\begin{aligned}
I & \approx 0.74\left(1-\sigma_{d}^{2}\right)+0.26\left(1-\sigma_{d}^{2}\right)^{2}, \\
\sigma_{d, k}^{2} & \approx 2.42-\sqrt{2.03+\frac{I_{E, k}^{\mathrm{TD}}}{0.26},}
\end{aligned}
$$

we get an expression for the effective mutual information for the extrinsic output of the $K$ TDs

$$
\begin{gathered}
I_{E, \text { eff }}^{\mathrm{TD}}=0.74\left[1-\sum_{k=1}^{L} \alpha_{k}^{*}\left(2.42-\sqrt{2.03+\frac{I_{E, k}^{\mathrm{TD}}}{0.26}}\right)\right] \\
+0.26\left[1-\sum_{k=1}^{L} \alpha_{k}^{*}\left(2.42-\sqrt{2.03+\frac{I_{E, k}^{\mathrm{TD}}}{0.26}}\right)\right]^{2} .
\end{gathered}
$$

Fig. 2 shows the EXIT characteristics from (2) after 6 iterations of the 3GPP compliant turbo decode TD for two groups where $P_{2}=2 P_{1}$ and $P_{\text {ref }}=P_{1}$, along with the effective EXIT curve in (5). We see that the EXIT curve for Group 2 is not simply an affine copy of that for Group 1 as it is in a variance transfer chart [4] since mutual information is not a linear function of variance. The IMUD receiver EXIT chart is shown in Fig. 3 for a loading of $\mathbf{K}=[20,20]$, spreading gain $N=40$, power $\mathbf{P}=[1,2]$ at $E_{b} / N_{0}=1.15 \mathrm{~dB}$ (where $E_{b}=P_{\text {ref }}$ ). 


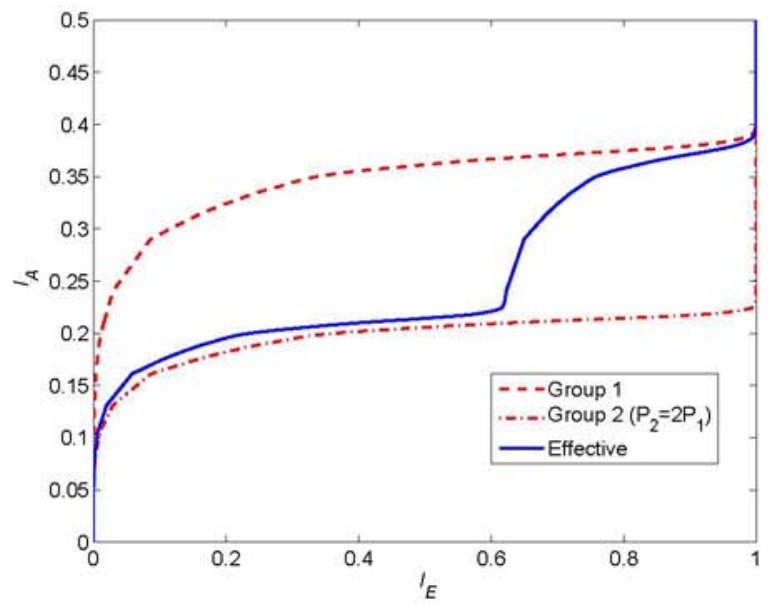

Fig. 2. EXIT chart for two user groups with a $3 \mathrm{~dB}$ power difference together with the corresponding effective EXIT chart.

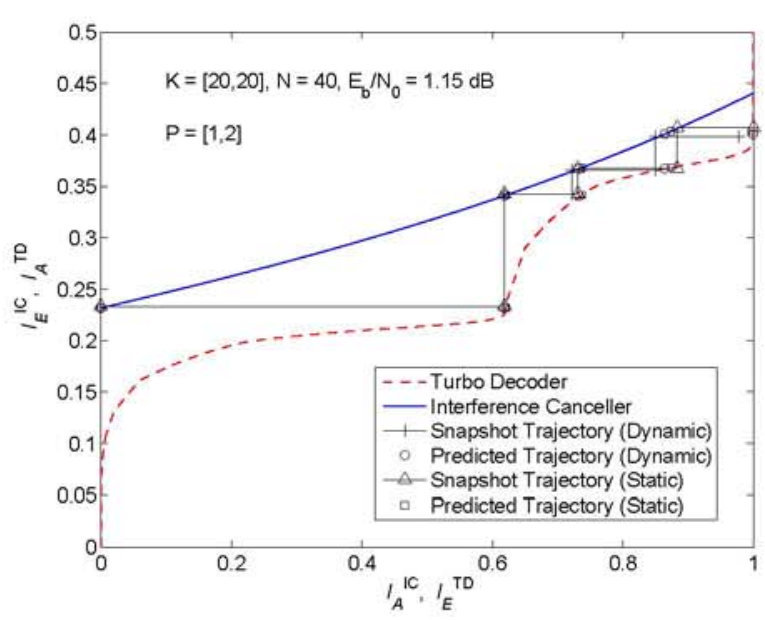

Fig. 3. EXIT chart showing predicted and simulated (snapshot) trajectories for the static and dynamic decoding schedules.

The effective number of users is $K_{\text {eff }}=60$ and this example system is used for all simulations in this paper. We define a decoder iteration as an iteration in the TD and a receiver iteration as an iteration between the interference canceller and the TDs. The predicted (squares) and simulated (triangles) trajectories are also shown in Fig. 3 for a static activation schedule with 4 receiver iterations, each running 6 TD iterations. We see that the simulation quite closely matches the EXIT chart analysis.

\section{OPTIMAL SCHEDULING}

The decoding complexity and delay are important practical considerations for the design of an IMUD receiver. Therefore, the optimal receiver decoding schedule is of interest. In

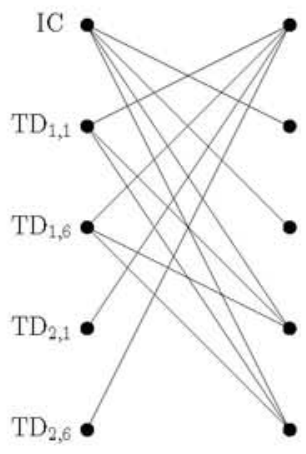

Fig. 4. Decoding trellis for two groups.

[5] the optimal scheduling for multiple concatenated codes is described. We extend this to the unequal power CDMA receiver, which is similar to a receiver for parallel concatenated codes in [5] with the difference that iterating between the TDs of different groups yields no gain in mutual information. This does not actually affect the derivation of the optimal schedule and the procedure applied in [5] can be used in the unequal power CDMA case.

In a conventional IMUD receiver decoding follows a fixed schedule. The optimisation algorithm described in [5] can be used to optimise the decoding schedule such that the decoding complexity and delay are minimized while a bit-error-rate (BER) performance is maintained. The algorithm is described below and utilizes a trellis and a modified Viterbi algorithm using mutual information as path metrics to predict the decoding performance of all relevant paths through the trellis. The algorithm differs from the Viterbi algorithm in that there can be multiple surviving paths into each state. A decoding trellis is shown in Fig. 4 for two groups where each group can run either 1 or 6 iterations of the TD. The subscripts in $\mathrm{TD}_{k, i}$ denote group $(k)$ and number of iterations $(i)$. Note that edges where no gain in mutual information is possible, such as a receiver block iterating with itself, have been removed.

\subsection{Definitions}

Let $m$ denote trellis transition and $L$ denote the number of groups (defined by power level). Each group is permitted $i \in$ $\left(1,2, \ldots, i_{\max }\right)$ iterations. Paths entering state $n$ are defined as $\mathbf{p}_{m}=\left(p_{1}, p_{2}, \ldots, p_{m}\right)$ where $p_{j} \in\left\{1,2, \ldots, i_{\max } L+1\right\}$ for $1 \leq j \leq m-1$ and $p_{m}=n$. The metric for the corresponding path is defined as

$$
\mathbf{v}=\left(\hat{P}_{b, 1}, \ldots, \hat{P}_{b, L}, C, I_{E, \text { eff }}^{\mathrm{IC}}, I_{E, \text { eff }}^{\mathrm{TD}}, I_{E, 1}^{\mathrm{TD}}, \ldots, I_{E, L}^{\mathrm{TD}}\right)
$$

where complexity $C$ is the total number of TD iterations. Complexity is updated as

$$
C_{m}=C_{m-1}+ \begin{cases}i & \text { for a turbo decoder activation } \\ 0 & \text { otherwise }\end{cases}
$$


where $i$ is the number of TD iterations. Here we make the assumption that the complexity of an interference canceller activation is insignificant in comparison to a TD iteration. Let $I_{D, k}$ denote the mutual information of the a posteriori output from TD group $k$. It can be calculated as

$$
I_{D, k}=J\left(\sqrt{J^{-1}\left(I_{A(s), k}^{\mathrm{TD}}\right)^{2}+J^{-1}\left(I_{E(s), k}^{\mathrm{TD}}\right)^{2}}\right)
$$

where $A(s)$ and $E(s)$ denote the a priori and extrinsic mutual information of the systematic bits, respectively. The expression in (8) can be used to estimate the BER of Group $k$ as [9]

$$
\hat{P}_{b, k}=Q\left(J^{-1}\left(I_{D, k}\right) / 2\right),
$$

which are the $L$ first elements in (6).

Define $\mathcal{P}_{m}$ and $\mathcal{V}_{m}$ as the sets of surviving paths and metrics respectively; and $\mathcal{P}_{m, n} \subseteq \mathcal{P}_{m}$ and $\mathcal{V}_{m, n} \subseteq \mathcal{V}_{m}$ as the sets of paths and metrics ending at state $n$ after $m$ trellis transitions. Define path $\mathrm{p}^{*}$ with metric $\mathrm{v}^{*}$ as the current (at transition $m$ ) optimal path. Define also the metric update function $f_{n}$ for each state $n$ [5], which updates all $2 L+3$ elements in $\mathrm{v}$ for all paths entering state $n$ using (9), (7), (1), (5), and (2).

Define domination as in [5], where metric $\mathbf{v}$ dominates $\mathbf{v}^{\prime}$ if and only if the extrinsic mutual information $v_{q}$ are higher than $v_{q}^{\prime}$ for $q=L+2, L+3, \ldots, 2 L+3$, respectively, and the complexity $v_{L+1}$ is less than or equal to $v_{L+1}^{\prime}$. Define target BER $P_{\text {target }}$ as the desired BER of each group of users.

\subsection{Algorithm}

The first step is using the results from Section 3 to derive the EXIT chart for the load/power/SNR configuration of interest. Fig. 3 shows the EXIT charts for an interference canceller and TDs under a system load described in Section 3.2. Then the intersection of the averaged EXIT curve (shown in Fig. 2) with the interference canceller curve determines the convergence point $I_{D}^{*}$.

The Viterbi search algorithm is as follows [5]

1. Let $m=1$. Initialize path set to contain only one path $\mathcal{P}_{m}=\{(1)\}$ and corresponding metric set $\mathcal{V}_{m}=$ $\left\{f_{1}(\mathbf{0})\right\}$. Initialize $\mathbf{p}^{*}=1$ and $v_{L+1}^{*}=\infty$.

2. $m=m+1$. For each state $n^{\prime}$ extend each path $p_{m-1}^{\prime}$ ending in state $n^{\prime}$ along the trellis defined transition $n^{\prime} \rightarrow n$, producing the new path $\mathbf{p}_{m}$ in $\mathcal{P}_{m, n}$, and update the metric in $\mathcal{V}_{m, n}$ using $\mathbf{v}=f_{n}\left(\mathbf{v}^{\prime}\right)$.

3. Remove all paths with complexity greater than or equal to that of the current optimal path $\mathrm{p}^{*}$.

4. Define a set of metrics $\mathcal{V}^{*}$ for paths that have reached the target BER $\left(v_{q} \leq P_{\text {target }}\right.$, for all $\left.q=1,2, \ldots, L\right)$ or the convergence point $I_{D}^{*}$. If there are multiple paths in $\mathcal{V}^{*}$ replace the candidate path $\mathrm{p}^{*}$ with the path of the lowest complexity.

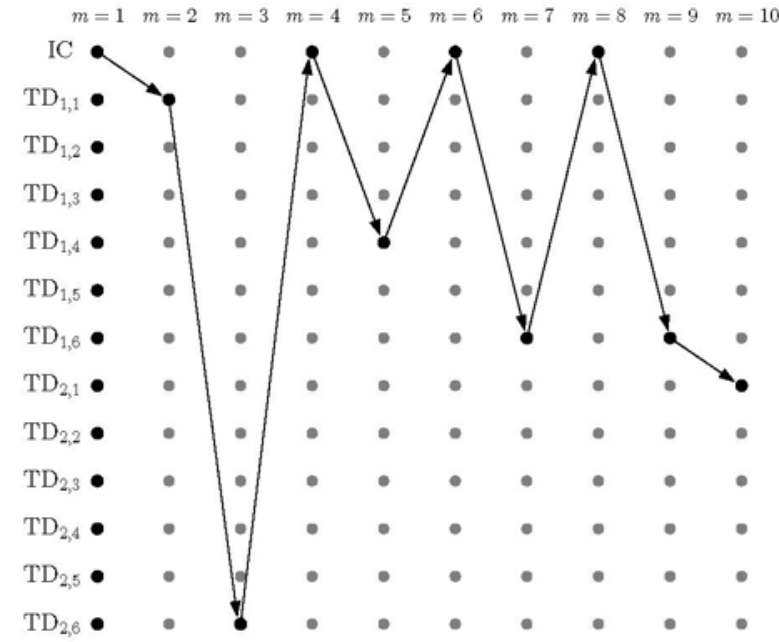

Fig. 5. Optimal path through the trellis to reach $P_{\text {target }}=10^{-4}$ for $\mathbf{K}=[20,20], \mathbf{P}=[1,2]$ at $E_{b} / N_{0}=1.15 \mathrm{~dB}$.

5. For each state, eliminate dominated metrics and their corresponding paths.

6. If no paths remain in $\mathcal{V}_{m}$ the candidate path $\mathrm{p}^{*}$ is the optimal path. Otherwise go to step 2.

The above algorithm could possibly result in an exponential growth of the number of surviving paths. However, as in [5], we have observed that in practice this exponential growth in $m$ does not occur and the number of surviving paths is small. Fig. 5 shows the optimum path (solid lines and arrow heads) through the trellis to reach $P_{\text {target }}=10^{-4}$ at $E_{b} / N_{0}=1.15 \mathrm{~dB}$. The notation is the same as in Fig. 4, i.e., the optimal schedule is: IC, 1 TD iteration of group 1, 6 TD iterations of group 2, IC, 4 TD iterations of group 1, IC, 6 TD iterations of group 1, IC, 6 TD iterations of group 1, and finally 1 TD iteration of group 2 . The corresponding predicted trajectory for the optimal schedule visualized through an EXIT chart is shown in Fig. 3 (circles).

\section{SIMULATION RESULTS AND DISCUSSION}

A turbo coded unequal power CDMA system was simulated using the conditions described in Section 3.2. We set the static decoding schedule as all groups running 6 TD iterations and 4 receiver iterations. Unless specified otherwise, all BER values are the system average, calculated as

$$
\hat{P}_{b}=\frac{1}{K} \sum_{k=1}^{L} K_{k} \hat{P}_{b, k} .
$$

The IMUD receiver was simulated for the static decoding schedule and the and dynamic decoding schedule derived in Section 4.2 and shown in Fig. 5. As suggested in [5], the optimal schedule when $P_{\text {target }}=10^{-4}$ is fulfilled (in this case 
at $E_{b} / N_{0}=1.15 \mathrm{~dB}$ as in Fig. 5) was chosen for all $E_{b} / N_{0}$ in the simulation. The corresponding EXIT chart snapshot trajectories are shown in Fig. 3. Both snapshot trajectories match quite closely with EXIT chart analysis. BER performance is plotted versus SNR in Fig. 6, the static schedule (squares) is shown for 1 to 4 receiver iterations and the dynamic schedule (crosses) is shown only after the 4 th receiver iteration. The static schedule BER performance of Group 1, $\hat{P}_{b, 1}$, is shown (dots) and for Group $2, \hat{P}_{b, 2}$ was less than $10^{-6}$ over the range of $E_{b} / N_{0}$ simulated. We see in Fig. 6 that the simulation results again quite closely match EXIT chart analysis (triangles and circles for static and dynamic schedules respectively) down to approximately $10^{-4}$ where we see an error floor in EXIT chart analysis since the target BER is fulfilled. More significantly, we see that BER performance of the dynamic schedule is very similar to that for the static decoding schedule while the dynamic schedule has a $50 \%$ reduction in complexity. That is, from Fig. 5 , for our given example the receiver requires only half the number of TD iterations (24 TD iterations for the dynamic decoding schedule compared to 48 TD iterations for the static decoding schedule) to achieve similar BER performance. A conventional receiver typically runs a high number of TD iterations and the dynamic schedule can drastically reduce the total decoding complexity as well as the delay. An IMUD receiver can therefore more efficiently use the physical resources if dynamic scheduling is used. That is, less TD iterations requires less power (clock speed can be reduced), and detection can be carried out successively through re-allocation of the physical resources to other users as they become available.

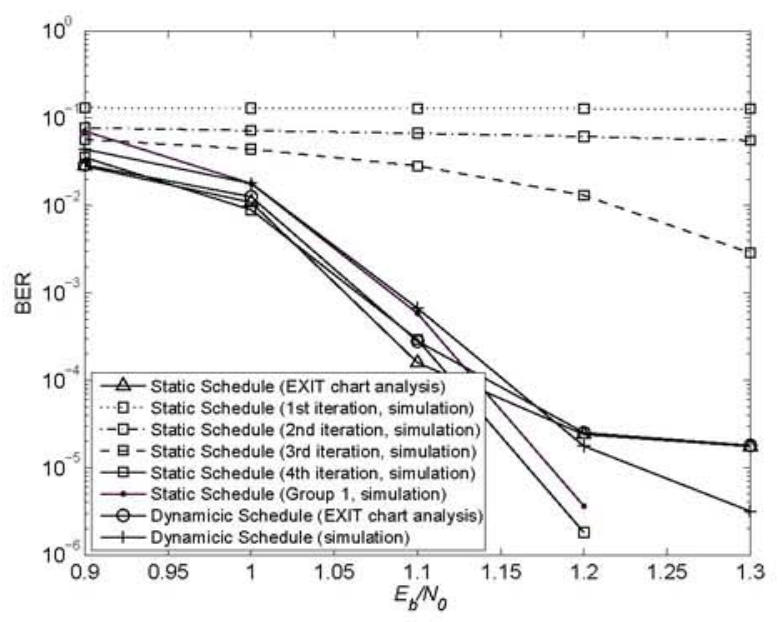

Fig. 6. BER performance of unequal power CDMA system for IMUD receiver following the optimal and static decoding (from Section 5) schedules.

\section{CONCLUSION}

We have investigated EXIT chart analysis of unequal power turbo-coded CDMA. The results in [4] were used to derive effective EXIT functions for FEC decoders and an interference canceller which enabled analysis of the system as in the equal power case. We used a modified version of the algorithm proposed in [5] to derive the optimal decoding schedule for the IMUD receiver and showed through simulation that this dynamic schedule achieves similar BER performance as a conventional receiver with significant complexity savings. Furthermore we showed that the effective EXIT chart analysis accurately predicts the convergence behavior of the system.

\section{REFERENCES}

[1] V. Kühn, "Analysis of iterative multi-user detection schemes with EXIT charts," in Proc. IEEE Eighth International Symposium on Spread Spectrum Techniques and Applications, September 2004, pp. 535-539.

[2] K. Li and X. Wang, "EXIT chart analysis of turbo multiuser detection," IEEE Transactions on Wireless Communications, vol. 4, no. 1, pp. 300-311, January 2005.

[3] G. Caire, R. R. Muller, and T. Tanaka, "Iterative multiuser joint decoding: optimal power allocation and low-complexity implementation," IEEE Trans. Info. Theory, vol. 50, no. 9, pp. 1950 1973, September 2004.

[4] Z. Shi and C. Schlegel, "Performance analysis of iterative detection for unequal power coded CDMA systems," in Proc. IEEE Globecom, December 2003, vol. 3, pp. 1537-1542.

[5] F. Brännström, L. K. Rasmussen, and A. J. Grant, "Convergence analysis and optimal scheduling for multiple concatenated codes," IEEE Trans. Info. Theory, vol. 51, pp. 3354-3364, September 2005.

[6] D. P. Shepherd, F. Brännström, and M. C. Reed, "Fidelity charts and stopping/termination criteria for iterative multiuser detection," to appear at 4 th International Symposium on Turbo Codes and Related Topics, April 2006.

[7] "3GPP TS 25.104 V5.9.0; 3rd generation partnership project;technical specification group radio access network; base station (BS) radio transmission and reception (FDD) (release 5)," September 2004.

[8] Paul D. Alexander, Alex J. Grant, and Mark C. Reed, "Performance analysis of an iterative decoder for code-division multiple-access," European Trans. on Telecom., vol. 9, no. 5, pp. 419-426, Sep./Oct. 1998.

[9] S. ten Brink, "Convergence behavior of iteratively decoded parallel concatenated codes," IEEE Trans. Commun., vol. 49, no. 10, pp. 1727-1737, Oct. 2001. 